\title{
The carnitine system and cancer metabolic plasticity
}

\author{
Mariarosa Anna Beatrice Melone ${ }^{1,2}$, Anna Valentino ${ }^{1,3}$, Sabrina Margarucci ${ }^{4}$, Umberto Galderisi ${ }^{5}$, \\ Antonio Giordano 2,6 and Gianfranco Peluso ${ }^{3}$
}

\begin{abstract}
Metabolic flexibility describes the ability of cells to respond or adapt its metabolism to support and enable rapid proliferation, continuous growth, and survival in hostile conditions. This dynamic character of the cellular metabolic network appears enhanced in cancer cells, in order to increase the adaptive phenotype and to maintain both viability and uncontrolled proliferation. Cancer cells can reprogram their metabolism to satisfy the energy as well as the biosynthetic intermediate request and to preserve their integrity from the harsh and hypoxic environment. Although several studies now recognize these reprogrammed activities as hallmarks of cancer, it remains unclear which are the pathways involved in regulating metabolic plasticity. Recent findings have suggested that carnitine system (CS) could be considered as a gridlock to finely trigger the metabolic flexibility of cancer cells. Indeed, the components of this system are involved in the bi-directional transport of acyl moieties from cytosol to mitochondria and vice versa, thus playing a fundamental role in tuning the switch between the glucose and fatty acid metabolism. Therefore, the CS regulation, at both enzymatic and epigenetic levels, plays a pivotal role in tumors, suggesting new druggable pathways for prevention and treatment of human cancer.
\end{abstract}

\section{Facts}

- Malignant cells are capable of creating an equilibrium between producing and consuming energy and metabolic intermediates synthesis to sustain growth and survival.

- Metabolic plasticity makes cancer cells more aggressive and able to metastasize. Oncogenic pathways, nutrient availability, and microenvironment influence cell metabolism.

Correspondence: Antonio Giordano (antonio.giordano@unisi.it) or Gianfranco Peluso (gianfranco.peluso@ibaf.cnr.it)

${ }^{1}$ Department of Medical, Surgical, Neurological, Metabolic Sciences, and Aging, 2nd Division of Neurology, Center for Rare Diseases and InterUniversity Center for Research in Neurosciences, University of Campania "Luigi Vanvitelli", Naples, Italy

${ }^{2}$ Department of Biology, Sbarro Institute for Cancer Research and Molecular Medicine, Center for Biotechnology, College of Science and Technology,

Temple University, Philadelphia, PA, USA

Full list of author information is available at the end of the article

These authors contributed equally: Melone Mariarosa Anna Beatrice and Valentino Anna.

Edited by A. Finazzi-Agrò
- The carnitine system is a pivotal mediator in cancer metabolic plasticity, intertwining key pathways, factors, and metabolites that supply an energetic and biosynthetic demand for cancer cells.

- MiRnas and metabolic enzymes regulate metabolic plasticity through the carnitine system suggesting their use for developing new therapeutic strategies.

\section{Open questions}

- What is the role of the carnitine system in cancer metabolism rewiring?

- Is the carnitine system dysregulated in cancerogenesis?

- What is the purpose of epigenetics in the modulation of the expression of proteins belonging to the carnitine system?

- Is it possible to explore new anticancer treatment targeting component(s) of the carnitine system? 
Cancer cells must maintain metabolic homeostasis in a wide range of conditions, including harsh microenvironments in which cancer cells must continue to meet the high bio-energetic demand in order to undergo replication $^{1}$. These cells achieve metabolic homeostasis by regulating the dynamics of nutrients present in the microenvironment, and the ability of cancer cells to utilize them to produce energy and to synthesize macromolecules. It is feasible that the capability of cancer cells to employ alternative nutrients in different environments is critical in supporting and affecting their survival. However, tumor cell metabolic plasticity is not just as the result of the metabolic dynamic impact changes induced by the microenvironment and fuel choices of cancer cells. Instead, it is more appropriate to envision metabolic plasticity regarding uptake of alternative metabolic substrates and promotion of metabolic rewiring as a built-in feature that has evolved to allow cancer cells to constantly adapt to changing intracellular- and extracellular metabolic conditions ${ }^{2}$.

Intracellular metabolite concentrations have to finetune the signaling networks governing metabolic pathways independently of the environment to ensure a balance between the availability of nutrients and the cellular capacity to use them effectively. Metabolites, through post-translational modifications of metabolite-sensitive protein (i.e. acetylation, methylation or glycosylation), transduce the information on the cell metabolic status, and modulate the activities of signaling proteins, enzymes, and transcriptional regulators ${ }^{3}$. Therefore, it is necessary to understand how a variety of intrinsic and extrinsic factors are integrated to create the metabolic flexibility and to reduce the metabolic dependencies dictated by oncogenic signaling. In this review, we identify the carnitine system (CS) as a gridlock to finely trigger the cancer cells' metabolic plasticity. In this context, the CS regulation at both the enzyme and the gene level plays a pivotal role in the metabolic flux modulation of tumors, and scientists can target them for therapeutic purposes.

\section{Nutrients and energy acquisition strategies in cancer cells metabolism}

Cancer cells prioritize aerobic glycolysis (Warburg effect), as the primary fuel and convert excess pyruvate to lactate independently from oxygen availability ${ }^{4}$. In addition, glucose is considered to be the primary carbon source that contributes to the production of mitochondrial citrate in cancer cells. The citrate in excess of mitochondrial requirements is exported in the cytosol, converted into acetyl-CoA by ATP-citrate lyase (ACLY), and used for protein acetylation and lipogenesis. While the principles regulating glucose-dependence in cancer cells have been extensively reviewed, we still do not fully understand how cancer cells use many of the metabolic strategies to contribute to core metabolic functions in the presence of nutrients different from glucose. Indeed, in addition to glycolysis, cancer cells can carry out various metabolic strategies such as fatty acid oxidation (FAO) ${ }^{2,5}$. Recent studies have reported that lipids from neighboring adipose tissues, lipoproteins, lysophospholipids, and intracellular storage fat have the potential to maintain viability and growth of cancer cells ${ }^{6-9}$. Fatty acids can satisfactorily fuel cancer cells, since mitochondrial FAO produces much more ATP per mole than oxidation of other nutrients, such as glucose or amino acids. For example, prostate cancer and B-cell lymphomas promote FAO as the main source of energy production and express FAO enzymes at high levels, even under nutrient-replete conditions $^{10,11}$. Again, autophagy and related processes enable tumor growth by sustaining oxidative phosphorylation ${ }^{12}$. Interestingly, the strong dependence of mitochondrial $\beta$-oxidation on autophagic and fatty acid (FA) catabolic processes makes some tumors more resistant to nutrient deprivation and environmental stressors ${ }^{13}$.

A peculiarity of several tumors is to present simultaneously two metabolic pathways in opposite directions, such as fatty acid biosynthesis and FAO ("futile cycle"). This paradoxical condition fulfils two fundamental tasks: (a) it provides the biosynthesis of FA important for cancer propagation, while ensuring an important source of ATP and Nicotinamide adenine dinucleotide (NADH) by the catabolism of any FA excess; and (b) it might induce a dynamic switching behavior that is useful in triggering signaling pathway(s) able to overcome metabolic stress maintaining cellular energy homeostasis (i.e. AMPactivated protein kinase; AMPK) ${ }^{14,15}$.

However, unlike glycolytic and lipogenic pathways, where specific metabolic enzymes such as hexokinase 2 and FAS are known to be deregulated by oncogene(s) or by inactivation of tumor suppressors ${ }^{16,17}$, there is limited evidence for cancer-associated abnormal expression or activity of the enzymes directly involved in the FAO pathway. Regarding expression and activity, the knowledge about the regulation of carriers and enzymes that modulate $\beta$-oxidation in cancer cells is of extreme interest since their inhibition may significantly affect the tumorigenic potential even in the presence of compensatory metabolic pathways.

\section{The carnitine system and its implication in cancer cells metabolic plasticity}

The use of diet-derived or adipose tissue-released longchain fatty acids as energy substrates requires about 25 different enzymes and transport proteins, which carry out fatty acids, import, them into mitochondria, and facilitate the $\beta$-oxidation steps. In particular, the mitochondrial inner membrane is impermeable to fatty acyl-CoA thioesters and, thus, the specialized CS, for transporting 
fatty acids across mitochondrial membranes has evolved $^{18}$. Components of the CS are both enzymes able to catalyze the acyl-CoA + carnitine $\leftrightarrow \mathrm{CoA}+$ acylcarnitines reaction and carrier(s) involved in the bi-directional transport of acyl moieties from cytosol to mitochondria and vice versa. Four components comprise this transporting system: the carnitine palmitoyltransferase 1 (CPT1) and 2 (CPT2), the carnitine-acylcarnitine translocase (CACT), and the carnitine acetyltransferase (CrAT) that close the carnitine cycle, allowing the export of the FAO-produced acetyl-CoA as acetyl-carnitine (Fig. 1).

\section{Carnitine palmitoyl transferase family Carnitine palmitoyltransferase $1 \mathrm{~A}$}

CPT1A is present mostly in the colon, duodenum, liver, kidney, and small intestine, and its deficiency results in a rare autosomal recessive metabolic disorder of long-chain $\mathrm{FAO}^{19}$. The overexpression of CPT1A is often associated with tumor progression in several cancers such as breast, gastric, prostate, lymphoma, leukemia, ovarian, lung, and myeloma $^{20-23}$. Several studies reported that inhibition/ depletion of CPT1 leads to apoptosis and suppression of cancer cell proliferation, chemoresistance, and neovascularization $^{24-28}$. It has been proposed that CPT1A contributes to cell survival, not only by increasing $\mathrm{FAO}^{29}$ but also by stimulating histone acetylase activity in the nucleus ${ }^{30}$. CPT1A can also protect cells from apoptosis by clearing the cytoplasmic long-chain fatty acyl-CoA such as palmitoyl-CoA, and thus impede the production of "palmitate/palmitoyl-CoA/ceramide" involved in the apoptosis pathway activation ${ }^{31}$. Pharmacological inhibition of CPT1A results in impaired cancer cell proliferation in acute myeloid leukemia and intensive cytotoxicity in Burkitt's lymphoma ${ }^{23,32}$. Interestingly, the CPT1 inhibition decreases $\beta$-oxidation, which remarkably attenuates c-myc-mediated lymphomagenesis. This suggests a potential role of CPT1 in the pathogenesis of c-mycdriven cancer. In particular, Ricciardi et al. demonstrated in vitro the anti-leukemic activity of the novel CPT1A reversible inhibitor ST1326 on leukemia cell lines and primary cells obtained from patients with hematologic malignancies, which induces cell growth arrest,

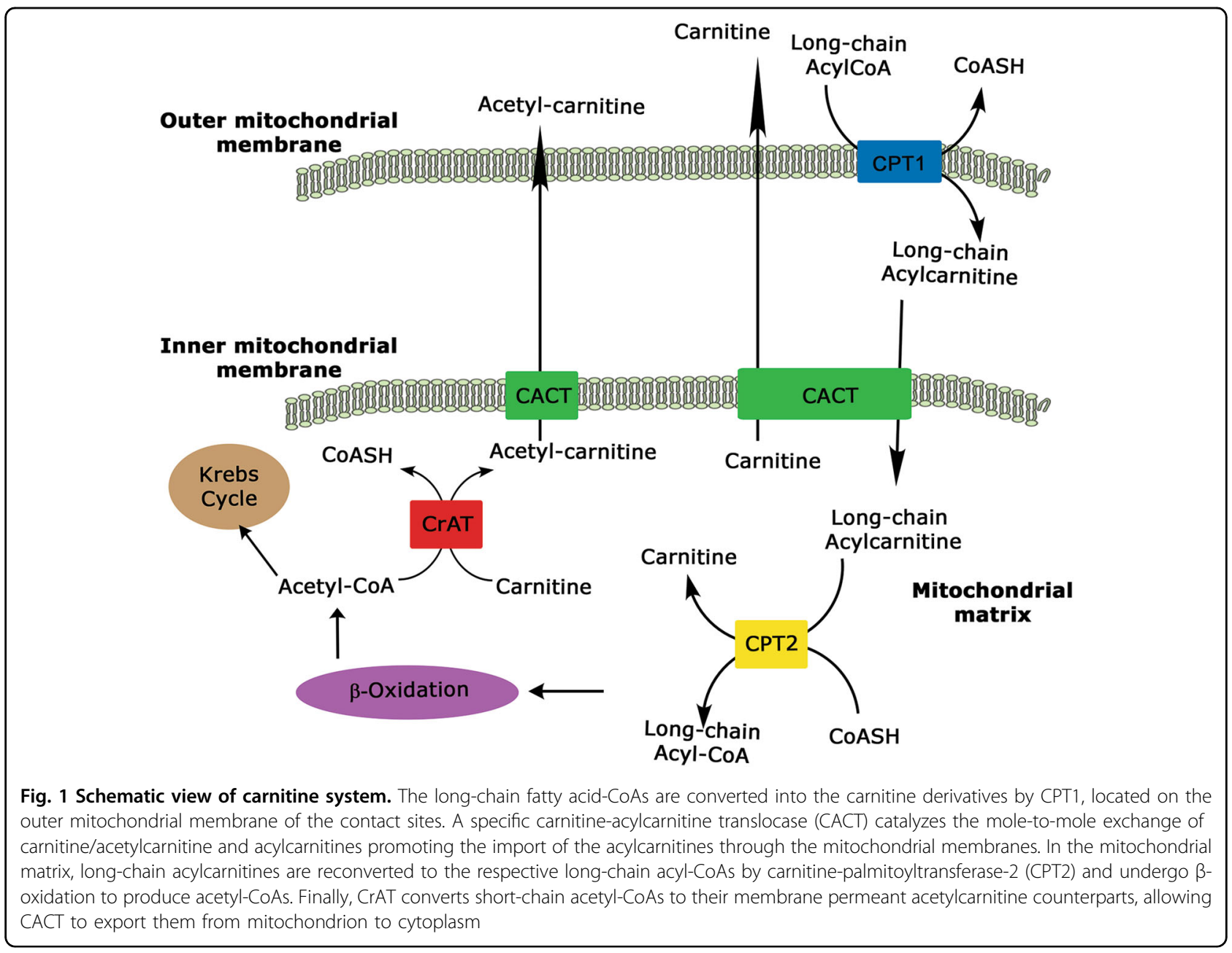


mitochondrial damage, and apoptosis ${ }^{32}$. Moreover, Pacilli et al. demonstrated that ST1326 inhibits FAO not only by blocking CPT1A but also by inhibiting CACT activity ${ }^{23}$. In addition, Shao et al. proved that most ovarian cancer cell lines express CPT1A highly and that its inactivation decreased cellular ATP levels, inducing cell cycle arrest at G0/G1 ${ }^{33}$. Several studies highlight the role of CPT1A in prostate cancer. Indeed, Schlaepfer et al. showed that the blockage of CPT1A (with etomoxir) and the lipid synthesis/lipolysis (with orlistat) decreased the viability of the androgen-dependent prostate cell lines LNCaP, VCaP, and patient-derived prostate cancer cells ${ }^{34}$. These effects were associated with decreased androgen receptors, mTOR and AKT expression signaling, and increased

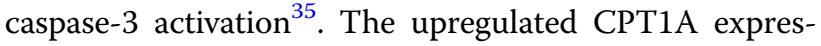
sion and activity was also observed in PC3 and LNCaP malignant prostate cells (androgen independent and dependent cells) and human prostate cancer specimens ${ }^{22}$. This recent study identifies an miRNA that targets CPT1A as a potential biomarker for therapy. Upregulated expression of CPT1A has also been determined in breast cancer by an integrated genomic strategy based on the use of gene expression signatures of oncogenic pathway activity $^{20}$. Moreover, Linher-Melville et al. showed that prolactin (PRL) increased the expression and the activity of CPT1A in breast cancer cells with respect to normal cells $^{29}$. The authors suggest that inhibition of FAO may lead to an overall decrease in cancer cell survival, while an increase in CPT1 activity, such as the PRL-mediated response, may provide a supportive environment for malignant cells ${ }^{36}$. Malonyl-CoA, the product of acetylCoA carboxylases (ACC), inhibits CPT1A. ACC exists as two related enzymes, the cytosolic isoform $\mathrm{ACC} 1$ and the mitochondrion-anchored ACC2. Both enzymes catalyze ATP-dependent carboxylation of acetyl-CoA to form malonyl-CoA. ACC1 is thought to be the predominant isoform mediating fatty acid synthesis, whereas ACC2, the isoform located near CPT1, is the major regulator of CPT1 activity. ACC1 expression has been shown to be frequently upregulated in several tumor types ${ }^{37}$, and have reported the expression and/or activation of ACC2 to be inhibited in a variety of cancers ${ }^{38}$.

\section{Carnitine palmitoyltransferase 1 B}

Carnitine palmitoyltransferase $1 \mathrm{~B}(\mathrm{CPT} 1 \mathrm{~B})$ is expressed mainly in tissues characterized by high rates of FAO, such as muscle and brown adipose tissue. CPT1B is also expressed in minor quantities in the testis, spleen, duodenum, lymph node, and stomach. Although CPT1A and CPT1B exhibit considerable sequence similarity, the sensitivity of these two enzymes to their physiological inhibitor, malonyl-CoA, differs greatly (CPT1A has a tenfold higher Ki for malonyl-CoA $)^{39,40}$. CPT1B is involved in human colorectal cancer as determined by Yeh et al. ${ }^{41}$.
By using a combined approach of MicroarrayBioinformatic technologies, the authors have revealed the overexpression of CPT1B in clinical tissue specimens of colorectal cancer, demonstrating a potential metabolic mechanism contributing to colorectal cancer. Moreover, recently, Kim et al., have demonstrated the deregulation of some carnitine-acylcarnitine metabolic pathwayassociated genes, such as CPT1B, that results in an increased mortality in patients with muscle-invasive bladder cancer ${ }^{42}$.

\section{Carnitine palmitoyltransferase $1 \mathrm{C}$}

The brain-specific carnitine palmitoyltransferase 1C (CPT1C) displays high-affinity to bind malonyl-CoA, but its enzymatic activity cannot be observed using conventional assays ${ }^{43}$. Recent studies showed that CPT1C was localized both in the endoplasmic reticulum and mitochondria, but its presence in endoplasmic reticulum is predominant. CPT1C is involved in cellular energysensing pathways and has an important role in the hypothalamic regulation of energy homeostasis ${ }^{44}$. It has been reported that the CPT1C isoform is overexpressed in human tumor cells such as neuroblastoma, several sarcomas of soft-tissues and lung, and malignant peripheral nerve sheath tumors (associated with neurofibromatosis type 1$)^{45}$. When CPT1C is upregulated, it increases FA consumption, and ATP production, facilitating tumor growth and survival ${ }^{43}$. Interestingly, depletion of CPT1C in mice led to delayed tumor development and a striking increase in survival ${ }^{46,47}$. Sanchez-Macedo et al. showed that p53 directly activated CPT1C transcription, conferring to tumor cells resistance ${ }^{46}$. A recent study indicates that CPT1C might be induced in the MCF-7 breast cancer cell line by the indirect action of activated $5^{\prime}$ AMPactivated protein kinase $(\text { AMPK })^{48}$. Moreover, Zaugg et al. showed that MCF-7 breast cancer cells constitutively overexpressing CPT1C displayed an increased FAOdependent ATP production, and resistance to glucose deprivation or hypoxia ${ }^{43}$. Finally, a recent study revealed that peroxisome proliferator-activated receptor alpha (PPAR $\alpha$ ) upregulated the expression of CPT1C, in a p-53 independent way, modulating proliferation and senescence of tumor cells ${ }^{49}$. These findings suggest that CPT1C is a regulator of FA homeostasis in cancer cells and might be indirectly involved in the modulation of CPT1A activity through the lowering of cellular malonyl-CoA levels. Indeed, $\mathrm{CPT} 1 \mathrm{C}$ binds to malonyl-CoA with the same affinity as CPT1A. Recently, in high-grade glioblastoma, Cirillo et al. showed an association between the expression of CPT1C and ZFP57, a zinc finger protein involved in gene imprinting ${ }^{50}$. Since a truncated form of CPT1C has been demonstrated in the nuclei of human diffuse gliomas, new perspectives are opening on the role of CPT1C expression in cancer cells ${ }^{51}$. 


\section{Carnitine palmitoyltransferase 2}

Compared to CPT1 isoforms, less is known about CPT2 deregulation in cancer; nevertheless, a recent study has reported that this enzyme could be considered as an independent prognostic factor in colorectal cancer patients ${ }^{52}$. Another study analyzed the expression of several enzymes involved in FA metabolism in the leukemia cells compared to normal cells. The results showed that leukemia cells presented a higher expression of most CPT isoforms, including CPT2. This suggests that chronic lymphocytic leukemia cells were highly active in de novo FA synthesis as well as in FA catabolism ${ }^{53}$. In addition, a recent paper correlates the phosphorylation state of Src with the expression and activity of CPT1A and CPT2 in triplenegative breast cancer $(\mathrm{TNBC})^{54}$. It is well known that aberrant Src activation plays prominent roles in cancer formation and progression ${ }^{55}$. The authors determined by trans-mitochondrial cybrids and multiple OMICs approaches that TNBC exhibit high levels of ATP through FAO and activates Src oncoprotein through autophosphorylation. The inhibition of FAO by the knocking down of CPT1 and CPT2 significantly decreased the phosphorylation level of $\mathrm{Src}$ and the number of metastatic nodules, confirming the role of mitochondrial FAO and CPT genes in Src regulation and their significance in breast cancer metastasis.

\section{Carnitine translocase and transferase components Carnitine/AcylCarnitine translocase}

Mitochondria import acylcarnitines through carnitine/ acylcarnitine translocase (CACT) located in the inner mitochondrial membrane, which catalyzes a mole-to-mole exchange of carnitines/acetylcarnitines and acylcarnitines $^{56}$. It has been demonstrated that, together with the regulation of CPT1 by malonyl-CoA, acetylation of CACT plays a crucial role in the control of the influx of fatty acyl units into mitochondria ${ }^{57}$. Peluso at al. hypothesized that in obesity-related insulin resistance (IR), skeletal muscle may decrease FAO because of decreased CACT activity ${ }^{58}$. Subsequently another work of the same research team analyzed the metabolic effects of CACT down-expression in human myocyte cells stably transfected with CACT antisense cDNA. This study revealed a complex metabolic situation determined by insulin and palmitate in CACTdeficient cells, and has provided insight into the functional interactions between CACT, CPT1, malonyl-CoA, and acetyl-carnitine ${ }^{59}$. To date, the correlation between CACT and cancer has received little attention, and only a few studies have reported a link between the altered expression of CACT and cancer. Kim et al. have demonstrated that in bladder cancer patients the expression of carnitine enzymes such as CACT has significantly deregulated in tumor tissues compared to normal bladder tissues ${ }^{42}$. Moreover, Valentino et al. demonstrated that in androgendependent and -independent prostate tumor cells as well as in human prostate cancer specimens, the overexpression and the increased activity of CACT is a hallmark of prostate cancer ${ }^{22}$.

\section{Carnitine $\mathbf{O}$-acetyltransferase}

Carnitine $\mathrm{O}$-acetyltransferase (CrAT), located primarily in the mitochondrial matrix, catalyzes the addition or the removal of carnitine from medium- and short-chain acyl$\mathrm{CoA}^{60,61}$, facilitating the efflux of mitochondrial acetyl$\mathrm{CoA}$ to the cytosol and buffering the intracellular pools of acetyl-CoA and carnitine. CrAT deficiency leads to accumulating acetyl-CoA, which exerts an allosteric inhibiting effect on pyruvate dehydrogenase (PDH), a rate-limiting enzyme for pyruvate entry into the Krebs cycle. This metabolic alteration has been associated with metabolic diseases or to insulin deficiency ${ }^{62-64}$. Interestingly, the CrAT activity is reduced during obesity and aging, leading to impaired glycemic control ${ }^{65}$. Studies in Crat knockout mice demonstrated that CrAT deficiency leads to abnormal fuel selection, which results in a perturbation of glucose homeostasis and suggest that deficits in CrAT activity might contribute to diet-induced metabolic inflexibility by exacerbating the Randle glucose-fatty acid cycle ${ }^{66}$. The higher CrAT amount determined both in prostate cancer cells (PC3 and LNCaP, androgen-dependent and androgen-independent, respectively) and prostate tumor biopsy by Valentino et al. highlight the importance of CrAT to contribute to maintaining a high metabolic plasticity of prostate cancers ${ }^{22}$. Finally, CrAT might play a fundamental role in histone acetylation in cancer cells. From a canonical point of view, ATP Citrate Lyase (ACL) produces acetyl-CoA from mitochondrion-derived citrate. Most of the acetyl-CoA produced by ACL originally derives from glucose or glutamine carbon. In addition to contributing to FA synthesis, acetyl-CoA can regulate cell growth by controlling the expression of genes involved in this process by histone acetylation. Alternatively, acetylcarnitine, produced in excess in mitochondria by CrAT, is transported into cytosol by CACT and enters the nucleus, where a nuclear CrAT converts the acetylcarnitine to acetyl-CoA ${ }^{67}$, and it becomes a source of acetyl groups for histone acetylation. Therefore, besides citrate-derived acetyl-CoA by nuclear ATP-citrate lyase ${ }^{68}$, the carnitine-mediated supply of acetyl groups is also an important source of acetyl-CoA for nuclear histone acetylation. Indeed, genetic deficiency of the translocase markedly reduced the mitochondrial acetylcarnitinedependent nuclear histone acetylation, indicating the significance of the carnitine-dependent mitochondrial acetyl group contribution to histone acetylation.

\section{Metabolic intermediates and carnitine system}

Metabolic pathways are monitored and controlled by allosteric or post-translational regulation of enzymes that 
catalyze specific biochemical reactions. Afterwards, alternative splicing, mRNA stability, translation, and protein degradation control the abundance of enzymes ("long-term regulation") ${ }^{69}$. In this view, nutrients, such as glucose or free fatty acids, provide intermediate metabolites the ability to interact directly with an enzyme (the metabolic sensor) that rules the rate limiting step of a metabolic process, thus regulating substrate preference. A case in point is the so-called "glucose-fatty acid cycle", a prime example of tightly coordinated cellular energy metabolism that provides a mechanistic reciprocal regulation of lipid and glucose oxidation to maintain cell homoeostasis and to avoid mitochondrial overloading. Interestingly, $\beta$-oxidation and aerobic glycolysis are connected to each other through cross-signaling in such a manner that beta-oxidation not only is compatible with ongoing aerobic glycolysis, but also it promotes the Warburg Effect. Indeed, the PDH complex, which decarboxylates glycolytically derived pyruvate to acetyl coenzyme A and links cytoplasmic glycolysis to the mitochondrial tricarboxylic acid (TCA) cycle, is modulated by reversible phosphorylation by PDH kinase (PDK). Nicotinamide adenine dinucleotide (NADH) and acetylCoA, two metabolic intermediates produced in the course of FAO, induce activation of PDK, which in turn phospho-inactivates the E1 $\alpha$ subunit of the PDH complex, leading to lower rates of glucose oxidation and higher rates of lactate release ${ }^{70}$. Interestingly, multiple transcription factors, such as Myc, Wnt, and hypoxia inducible factors, can cause a transcriptional increase of one or more PDK isoforms in a cancer cell. A cross-talk between metabolic intermediates produced by aerobic glycolysis, and enzymes belonging to the carnitine cycle is also present in cancer cells. The relationship between lactate and cancer growth reflects the pleotropic actions of this molecule able to influence the metabolic phenotype of the cancer cells. A recent study has demonstrated that lactateinduced acidification of the microenvironment over a period of weeks induces a metabolic adaptation of the tumor cell population, promoting $\beta$-oxidation as a

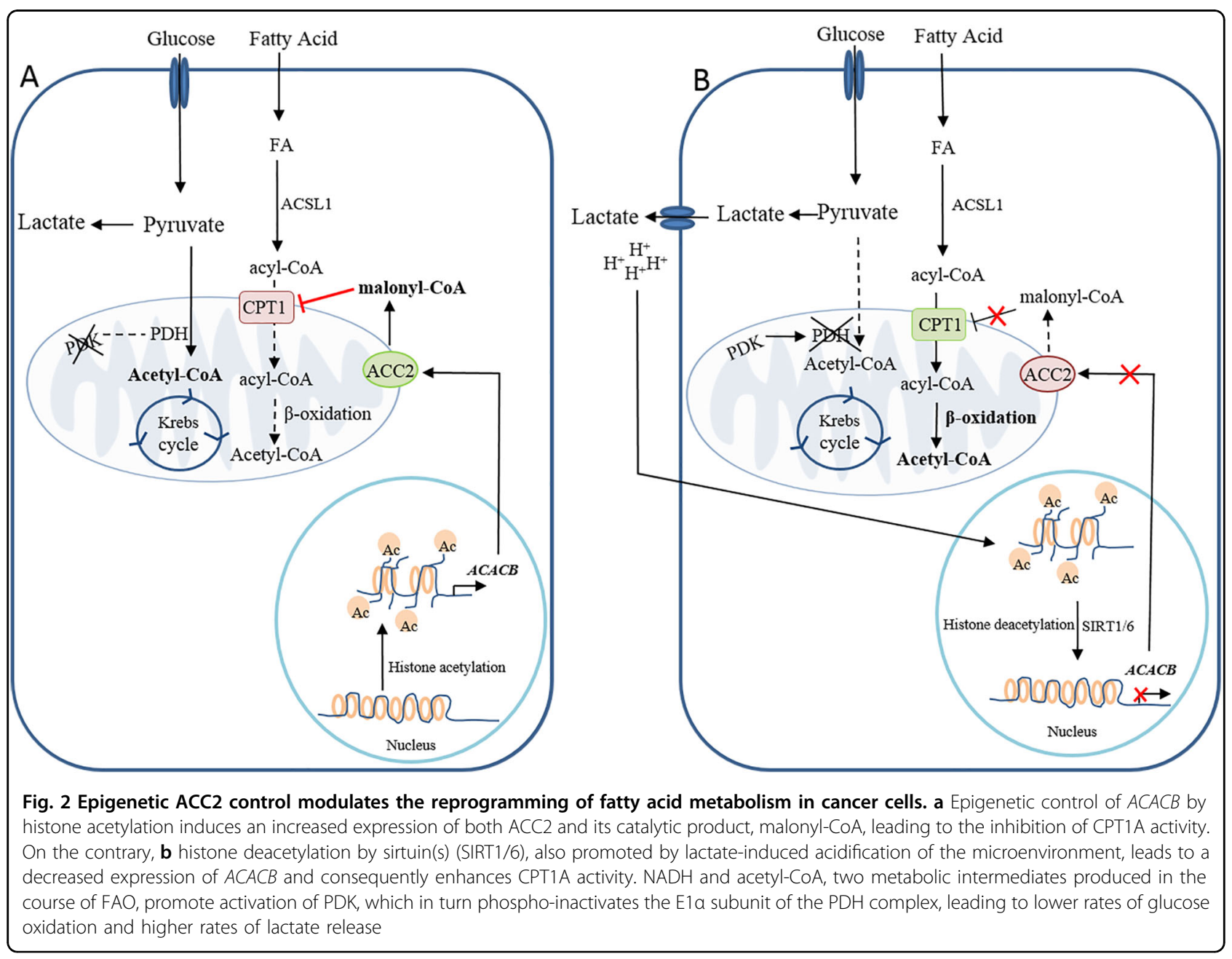


metabolic strategy (the Corbet-Feron Effect) ${ }^{71}$. In addition, cancer cells chronically exposed to an acidic $\mathrm{pH}$ reveal a downregulation of the mitochondrial ACC2 isoform, the enzyme that regulates CPT1 activity by malonyl-CoA synthesis. Therefore, only ACC1 isoform is expressed in tumor cells under acidosis to produce malonyl-CoA as a substrate for FA synthesis. Of interest, the down-expression of $\mathrm{ACC} 2$ results from an epigenetic process linked to histone deacetylation at the $\mathrm{ACACB}$ promoter by Sirtuin $1 / 6$ that are activated by high cytosolic $\mathrm{NAD}^{+}$levels associated with enhanced FAO and reduced glucose metabolism.

The non-enzymatic mitochondrial protein hyperacetylation induced by the increased availability of mitochondrial acetyl-CoA avoids the risk associated with mitochondria overfeeding, restraining the activity of the respiratory complex I and modulating CACT activity. Indeed, it has been demonstrated that acetylation plays a key role in the control of CACT function that, together with the long-chain acyl-CoA dehydrogenase, contributes to $\beta$-oxidation regulation. CACT acetylation compromises its activity, causing a decrease in its transport function. This mechanism represents a control of the influx of fatty acyl units into mitochondria in response to the intramitochondrial acetyl-CoA level, in addition to the finely regulated mechanism of CPT1 by malonyl-CoA. CACT acetylation mechanism is in line with a dynamic posttranslational protein control that could undergo an on/off switch induced by deacetylation/acetylation linked to the availability of acetyl-CoA ${ }^{57}$.

Recently, it was found that FA-derived acetyl-CoA is a significant font of carbon for acetylation of certain histone lysine, even in the presence of glucose ${ }^{72}$. Again, CS might be fundamental not only to produce but also to transport acetyl-CoA from the mitochondria to the nucleus for supplying acetyl-CoA for histone acetylation. Acetylcarnitine synthetized in mitochondria by CrAT is transported out mitochondria by CACT and transformed back to acetyl-CoA by CrAT present in the nucleus. In this model CS assumes a mechanistic role by which FA are perceived and integrated into the epigenome.

All these considerations are schematized in Figs. 2 and 3.

\section{Small-non-coding RNA (miRNAs) as a carnitine system regulator}

Among all small non-coding RNAs, microRNAs (miRNAs) (Fig. 4), 18-25 nucleotides in length, are well characterized as post-transcriptional regulators of mRNAs $^{73,74}$ and are considered a potential source of biomarkers for many diseases, including cancer ${ }^{75}$.

MiRNAs play important regulatory roles in a variety of biological processes such as cell proliferation, intercellular

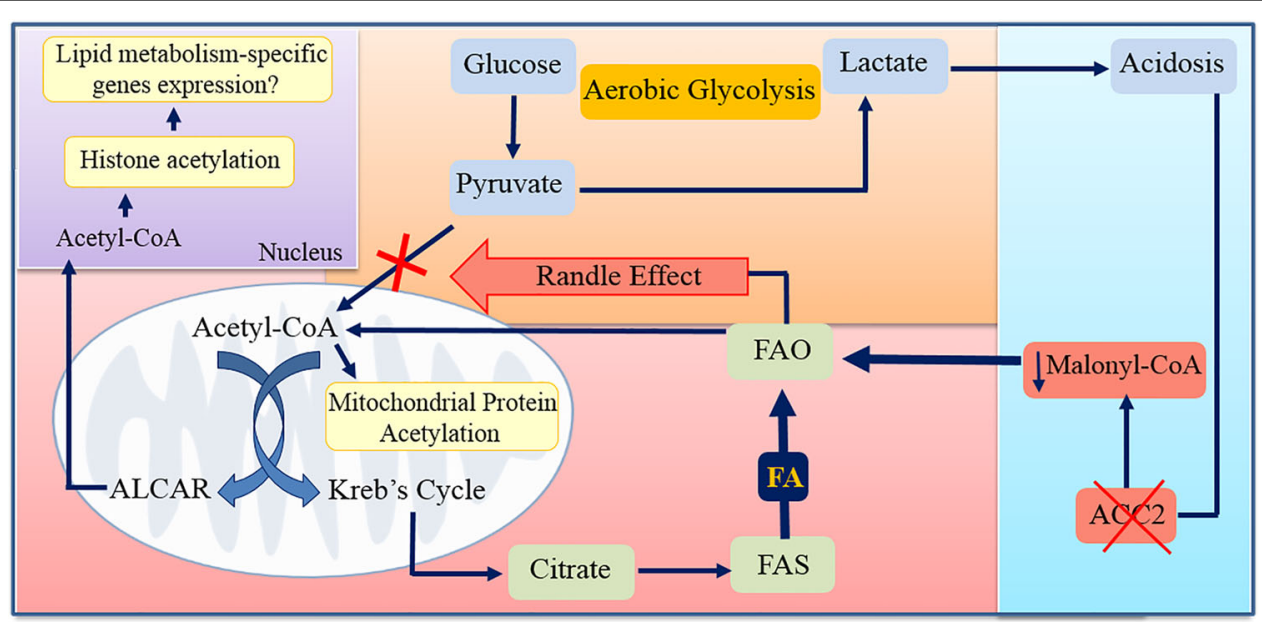

Fig. 3 The involvement of carnitine cycle in cancer cell metabolism. In cancer metabolism the aerobic glycolysis induces the conversion of the pyruvate (the end product of the glycolysis) into lactate (shown in orange) leading to the acidification of the microenvironment. The increased acidosis (shown in blue), in association with epigenetic mechanism(s), promotes the downregulation of the mitochondrial ACC2 isoform that in turn increases FAO (shown in red) via CPT1A upregulation. NADH and acetyl-CoA, produced in excess during FAO, promote the conversion of pyruvate into lactate through the inhibition of PDH by PDK (Randle effect). The increased availability of mitochondrial acetyl-CoA enhances the intramitochondrial non-enzymatic acetylation of proteins both of the Kreb's cycle and of the carnitine cycle, avoiding the risk associated with mitochondria overfeeding. In addition, the excess of acetyl-CoA is exported in the cytosol either as citrate or as acetyl L-carnitine (ALCAR). The citrate is converted into acetyl-COA by ACLY for the synthesis of fatty acids that might be re-imported into the mitochondria for beta-oxidation (Futile Cycle). ALCAR, shuttled to the nucleus (shown in violet), can be used as source of acetyl groups for histone acetylation, probably contributing to lipid metabolism-specific gene expression 


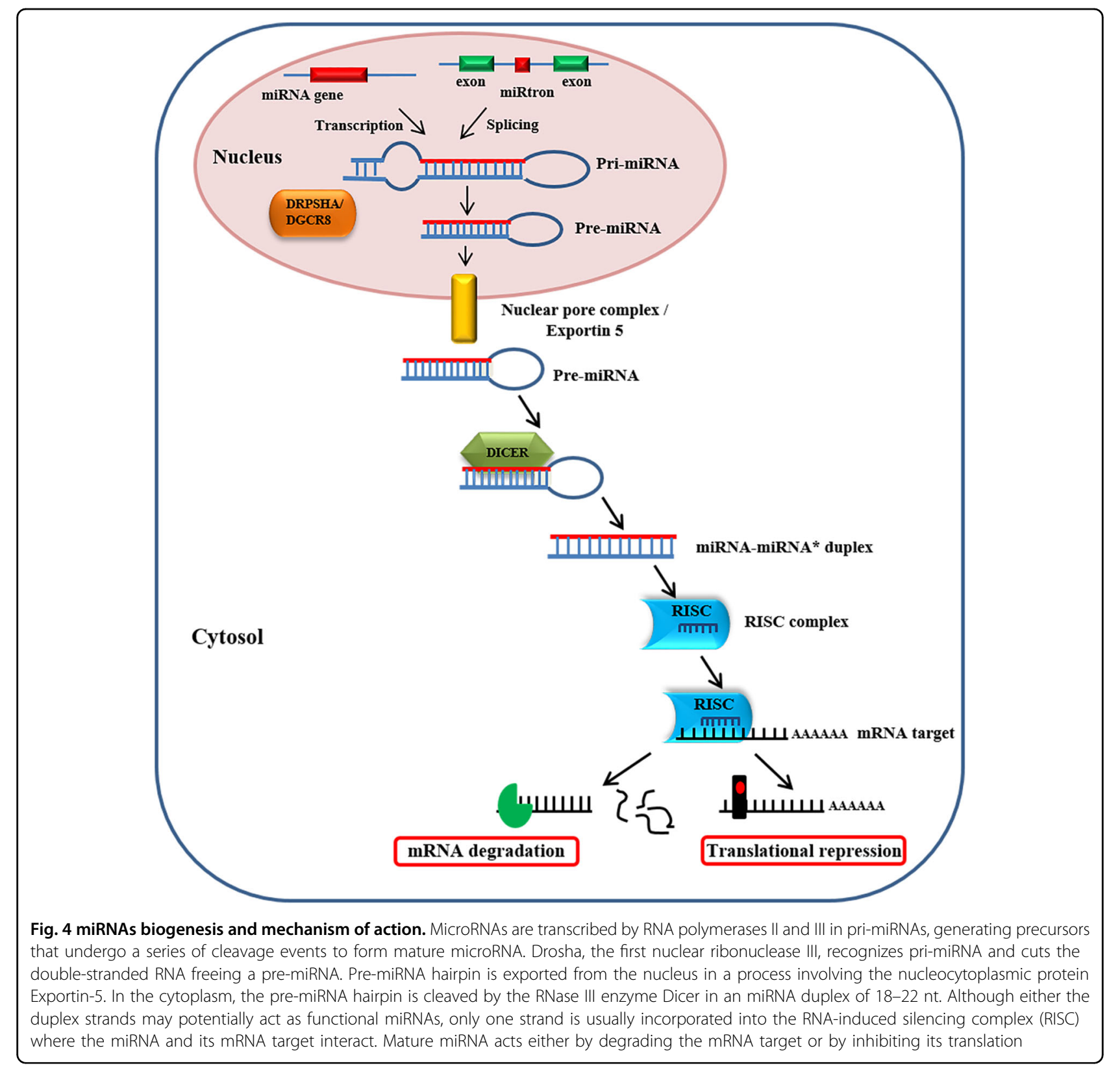

signaling, cell growth, cell death, cellular differentiation, apoptosis, and cancer metabolism ${ }^{76-78}$. Their expression profiles have been found to be tissue type-specific and frequently deregulated in various cancers ${ }^{79}$. MiRNAs have unique characteristics (i.e. stability, tissue specificity, easily detected and manipulated) that make them potential therapeutic targets for cancer treatment ${ }^{80}$. Emerging evidence demonstrates that miRNAs are critical regulators of lipid synthesis and $\mathrm{FAO}^{81}$ resulting in defective cell metabolism and carcinogenesis ${ }^{82}$ directly targeting key enzymes or transcription factors as oncogenes and tumor suppressors ${ }^{81}$ as shown in Table 1.
MiR-122 was the first miRNA identified as tissue-specific, and it is the most abundant in liver involved in lipid metabolic reprogramming ${ }^{83}$. MiR-122 inhibition leads to decreased plasma cholesterol and triglyceride levels associated with altered lipids biosynthesis and increased FAO. Several genes involved in fatty acid synthesis and oxidation were altered in mice treated with anti-miR-122 including FAS, $\mathrm{ACC} 1$, and $\mathrm{ACC} 2^{84}$. Furthermore, miR122 silencing in high-fat-fed mice reduced hepatic steatosis, with a decrease in cholesterol synthesis and stimulation of $\mathrm{FAO}^{85}$. Recently, Iliopoulos et al. identified a new miRNA, miR-370, that has effects on lipid metabolism 
Table 1 miRNAs involved in cancer metabolic plasticity

\begin{tabular}{lll}
\hline MiRNAs & Target & Reference \\
\hline miR-122 & Cholesterol biosynthesis & $88-90$ \\
miR-370 & Fatty acid oxidation, CPT1A & 91 \\
miR-378/378* & Lipid metabolism, CrAT & 92,93 \\
miR-335 & Lipid metabolism and adipogenesis & 94 \\
miR-205 & Lipid metabolism & 95 \\
miR-143 & Adipocyte differentiation & 96 \\
miR-27 & Adipolysis & 97 \\
miR-33a/b & Cholesterol efflux and $\beta$-oxidation & $98-100$ \\
miR-185 & Lipogenesis and cholesterogenesis & 101 \\
miR-342 & Lipogenesis and cholesterogenesis & 101 \\
miR-124 & CPT1A & 27 \\
miR-129 & CACT & 27,102 \\
\hline
\end{tabular}

similar to miR-122. MiR-370 targets CPT1A reducing FAO. Particularly, the human hepatic cell line HepG2 transfection with miR-370 upregulates the expression of miR-122. This upregulation leads to an increased expression of lipogenic genes, including sterol regulatory element-binding proteins (SREBP1c) and diacylglycerol acyltransferase-2 (DGAT2), which suggests that miR-370 provides a further point of regulation of this pathway ${ }^{86}$. Interestingly, components of cholesterol efflux and fatty acid metabolism are regulated by miR-33a and miR-33b. These miRNAs reside in intronic regions within SREBP-1 and 2, the key transcriptional regulators of lipid metabolism $^{87}$, and control the expression of carnitine $\mathrm{O}$ octanoyltransferase (CROT), CPT1A, hydroxyacyl coenzyme A (hydroxyacyl-CoA) dehydrogenase subunit beta (HADHB), and AMPK, targeting their $3^{\prime} \mathrm{UTR}^{88}$. Another important miRNA regulating cell metabolism is miR-378/ $378^{*}$, embedded within gene encoding peroxisome proliferator-activated receptor gamma coactivator 1-beta (PGC-1 $\beta$ ), a transcriptional regulator of oxidative energy metabolism. In breast cancer cells, a high level of miR$378^{*}$ induces the metabolic shift from an oxidative to a glycolytic bioenergetics pathway by inhibiting the expression of two PGC- $1 \beta$ partners, ERR $\gamma$ (estrogenrelated receptor gamma) and GABPA (GA binding protein transcription factor, alpha subunit). This leads to a reduction in TCA cycle gene expression and oxygen consumption as well as an increase in lactate production and cell proliferation ${ }^{89}$. Targets of miR-378 are also CRAT; indeed, it has been shown that mice genetically lacking miR-378 and miR-378* are resistant to high-fatdiet-induced obesity and display enhanced mitochondrial FA metabolism and elevated oxidative capacity of insulin- target tissue ${ }^{90}$. Moreover, Valentino et al. have demonstrated that the downregulation of hsa-miR-124-3p, hsamiR-129-5p, and hsa-miR-378 induces an increase in both expression and activity of CPT1A, CACT, and CrAT in malignant prostate cells ${ }^{22}$. In addition, the analysis of human prostate cancer and prostate control specimens confirmed the aberrant expression of miR-124-3p, miR129-5p, and miR-378 in primary tumors. Forced expression of the above-mentioned miRNAs affected tumorigenic properties, (i.e. proliferation, migration, and invasion), in PC3 and LNCaP cells regardless of their hormone sensitivity. MiR-143, miR-27, miR-335, miR-14, and miR-205 have been recently associated with lipid metabolism and adipocyte differentiation ${ }^{91}$. MiR-27a inhibits the expression of several lipid metabolic genes, including SREBP1-2, FASN, and PPAR $\alpha / \gamma$, by reducing lipid synthesis and increasing lipid secretion from cells ${ }^{92}$. The expression of MiR-335 is modulated by lipid loading, resulting in overexpression in liver and adipose tissue of obese mice ${ }^{93}$. However, the role of miR-335 in triggering lipid metabolism still remains unknown. Another miRNA implicated in the regulation of lipid metabolism is miR14. $\mathrm{Zu}$ et al., indeed, demonstrated in Drosophila melanogaster that the deletion of miR-14 increased the levels of triacylglycerol and diacylglycerol while its overexpression resulted in the converse effect ${ }^{94}$. Recently, it has been found that miR-205 deregulates lipid metabolism in hepatocellular carcinoma by targeting acyl-CoA synthetase ACSL1, a lipid metabolism enzyme in liver ${ }^{95}$. Furthermore, in prostate cancer cells, miR-185 and miR342 control lipogenesis and cholesterogenesis by reducing the expression of SREBP-1/2 and downregulating their targeted genes, including fatty acid synthase ${ }^{96}$. The function of miRNAs on lipid metabolic plasticity reveals molecular strategies aimed to control metabolic flux by miRNAs in cancerogenesis, thus lighting up one of miRNAs therapeutic aspect ${ }^{97}$ (Fig. 5).

\section{Concluding remarks}

Cancer metabolic plasticity allows tumor cells to survive in the face of adverse environmental conditions. Metabolites involved in metabolic plasticity must be able to fluctuate in response to internal or external perturbations. Therefore, identifying regulatory nodes within the metabolic network is challenging due to its complex structure. Although today several studies have investigated the mechanism involved in metabolic plasticity, but key pathway has not been identified which could be considered a unique target for cancer therapy. We critically review how cancer cells control the metabolic regulatory mechanisms. Therefore, we have emphasized the crucial role of the CS and its fine-tuned regulation, which is both enzymatic- and miRNA-dependent, as the primary factor in metabolic cancer flexibility. 


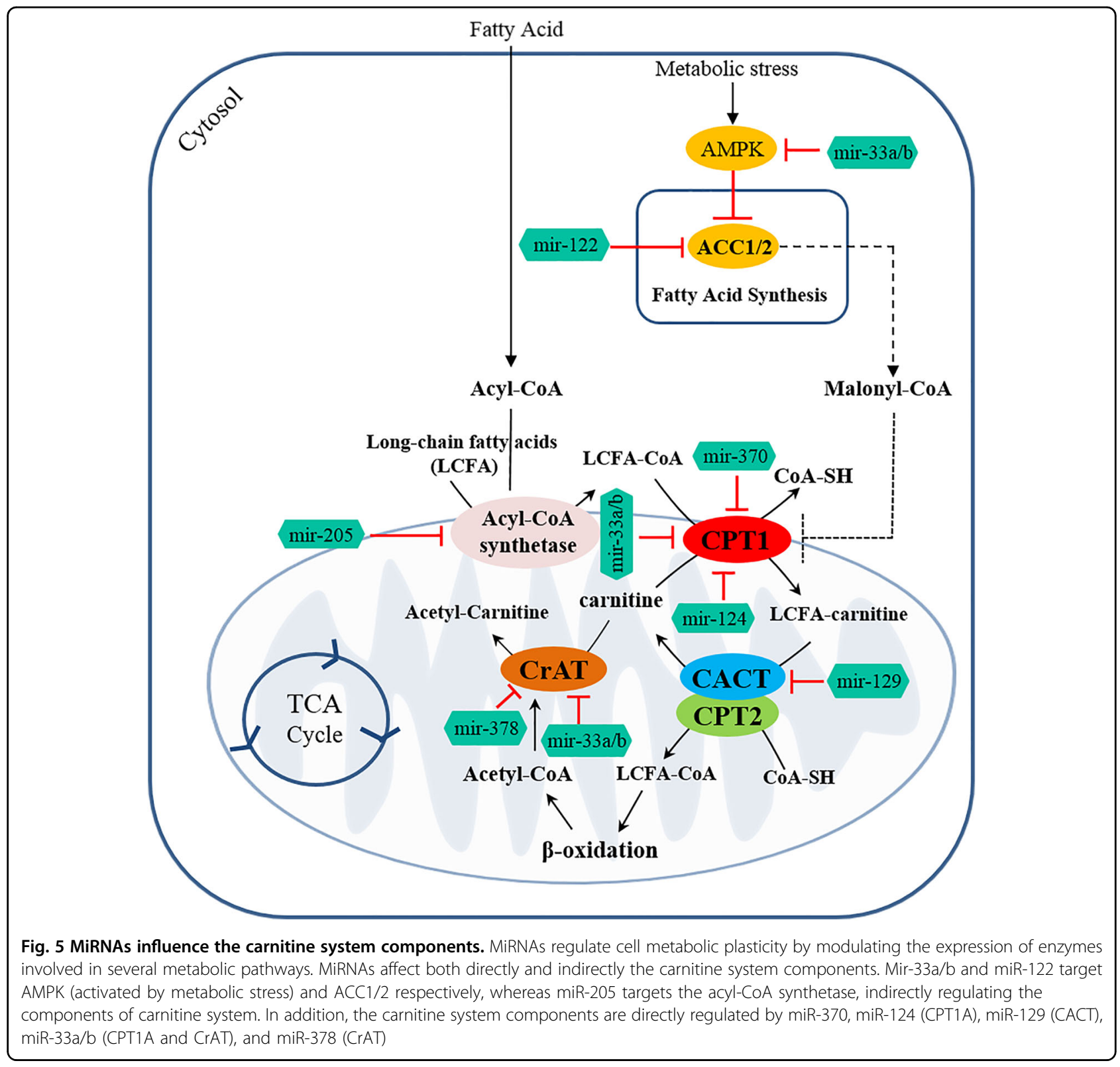

\section{Acknowledgements}

This work was supported by Ministero dell'Itruzione, dell'Università e della Ricerca of Italy, Progetto PON_-'Ricerca e Competitività 2007-2013' PON01_01802: Sviluppo di molecole capaci di modulare vie metaboliche intracellulari redoxsensibili per la prevenzione e la cura di patologie infettive, tumorali, neurodegenerative e loro delivery mediante piattaforme nano tecnologiche', and PON01_02512: 'Ricerca e sviluppo di bioregolatori attivi sui meccanismi epigenetici dei processi infiammatori nelle malattie croniche e degenerative'.

\section{Author details}

'Department of Medical, Surgical, Neurological, Metabolic Sciences, and Aging, 2nd Division of Neurology, Center for Rare Diseases and InterUniversity Center for Research in Neurosciences, University of Campania "Luigi Vanvitelli", Naples, Italy. ${ }^{2}$ Department of Biology, Sbarro Institute for Cancer Research and Molecular Medicine, Center for Biotechnology, College of Science and Technology, Temple University, Philadelphia, PA, USA. ${ }^{3}$ Institute of AgroEnvironmental and Forest Biology, National Research Council, IBAF-CNR,
Naples, Italy. ${ }^{4}$ Institute of Bioscience and BioResources, IBBR- CNR, Naples, Italy. ${ }^{5}$ Department of Experimental Medicine, Biotechnology and Molecular Biology Section, University of Campania "Luigi Vanvitelli", Naples, Italy. ${ }^{6}$ Department of Medicine, Surgery and Neuroscience, University of Siena, Siena, Italy

\section{Conflict of interest}

The authors declare that they have no conflict of interest.

\section{Publisher's note}

Springer Nature remains neutral with regard to jurisdictional claims in published maps and institutional affiliations.

Received: 5 December 2017 Revised: 9 January 2018 Accepted: 11 January 2018

Published online: 14 February 2018 


\section{References}

1. Goodpaster, B. H. \& Sparks, L. M. Metabolic flexibility in health and disease. Cell Metab. 25, 1027-1036 (2017).

2. Quail, D. \& Joyce, J. Microenvironmental regulation of tumor progression and metastasis. Nat. Med. 19, 1423-1437 (2013).

3. Pavlova, N. N. \& Thompson, C. B. The emerging hallmarks of cancer metabolism. Cell Metab. 23, 27-47 (2016)

4. Martinez-Reyes, I. et al. TCA cycle and mitochondrial membrane potential are necessary for diverse biological functions. Mol. Cell 61, 199-209 (2016).

5. Gao, X., Reid, M. A., Kong, M. \& Locasale, J. W. Metabolic interactions with cancer epigenetics. Mol. Asp. Med. 54, 50-57 (2016)

6. Nieman, K. M. et al. Adipocytes promote ovarian cancer metastasis and provide energy for rapid tumor growth. Nat. Med. 17, 1498-U207 (2011).

7. Rios, M. et al. Lipoprotein internalisation induced by oncogenic AMPK activation is essential to maintain glioblastoma cell growth. Eur. J. Cancer $\mathbf{5 0}$ 3187-3197 (2014).

8. Kamphorst, J. J. et al. Hypoxic and Ras-transformed cells support growth by scavenging unsaturated fatty acids from lysophospholipids. Proc. Natl. Acad. Sci. USA 110, 8882-8887 (2013).

9. Nomura, D. K. et al. Monoacylglycerol lipase regulates a fatty acid network that promotes cancer pathogenesis. Cell 140, 49-61 (2010).

10. Caro, P. et al. Metabolic signatures uncover distinct targets in molecular subsets of diffuse large B cell lymphoma. Cancer Cell 22, 547-560 (2012).

11. Wu, X., Daniels, G., Lee, P. \& Monaco, M. E. Lipid metabolism in prostate cancer. Am. J. Clin. Exp. Urol. 2, 111-120 (2014).

12. Yang, $\mathrm{S}$. et al. Pancreatic cancers require autophagy for tumor growth. Genes Dev. 25, 717-729 (2011).

13. Viale, A. et al. Oncogene ablation-resistant pancreatic cancer cells depend on mitochondrial function. Nature 514, 628-632 (2014).

14. Samoilov, M., Plyasunov, S. \& Arkin, A. P. Stochastic amplification and signaling in enzymatic futile cycles through noise-induced bistability with oscillations. Proc. Natl. Acad. Sci. USA 102, 2310-2315 (2005).

15. Hardie, D. G. \& Pan, D. A. Regulation of fatty acid synthesis and oxidation by the AMP-activated protein kinase. Biochem. Soc. Trans. 30(Pt 6), 1064-1070 (2002).

16. Alvarez, J. V. et al. Oncogene pathway activation in mammary tumors dictates FDG-PET uptake. Cancer Res. 74, 7583-7598 (2014).

17. Yoon, S. et al. Up-regulation of acetyl-CoA carboxylase alpha and fatty acid synthase by human epidermal growth factor receptor 2 at the translational level in breast cancer cells. J. Biol. Chem. 282, 26122-26131 (2007).

18. Bastin, J. Regulation of mitochondrial fatty acid beta-oxidation in human: what can we learn from inborn fatty acid beta-oxidation deficiencies? Biochimie 96, 113-120 (2014)

19. Longo, N., Amat di San Filippo, C. \& Pasquali, M. Disorders of carnitine transport and the carnitine cycle. Am. J. Med. Genet. C. Semin. Med. Genet. 142C, 77-85 (2006)

20. Gatza, M. L., Silva, G. O., Parker, J. S., Fan, C. \& Perou, C. M. An integrated genomics approach identifies drivers of proliferation in luminal-subtype human breast cancer. Nat. Genet. 46, 1051-1059 (2014).

21. Li, S. et al. High metastaticgastric and breast cancer cells consume oleic acid in an AMPK dependent manner. PLoS ONE 9, e97330 (2014).

22. Valentino, A. et al. Deregulation of MicroRNAs mediated control of carnitine cycle in prostate cancer: molecular basis and pathophysiological consequences. Oncogene 36, 6030-6040 (2017).

23. Pacilli, A. et al. Carnitine-acyltransferase system inhibition, cancer cell death, and prevention of Myc-induced lymphomagenesis. J. Natl. Cancer Inst. 105, 489-498 (2013).

24. Li, J. et al. Inhibition of lipolysis by mercaptoacetate and etomoxir specifically sensitize drug-resistant lung adenocarcinoma cell to paclitaxel. PLOS ONE $\mathbf{8}$, e74623 (2013).

25. Tung, S. et al. PPARalpha and fatty acid oxidation mediate glucocorticoid resistance in chronic lymphocytic leukemia. Blood 122, 969-980 (2013).

26. Schoors, S. et al. Fatty acid carbon is essential for dNTP synthesis in endothelial cells (vol 520, pg 192, 2015). Nature 526, 144 (2015).

27. Patella, F. et al. Proteomics-based metabolic modeling reveals that fatty acid oxidation (FAO) controls endothelial cell (EC) permeability. Mol. Cell Proteom. 14, 621-634 (2015)

28. Giordano, A. et al. tBid induces alterations of mitochondrial fatty acid oxidation flux by malonyl-CoA-independent inhibition of carnitine palmitoyltransferase-1. Cell Death Differ. 12, 603-613 (2005).
29. Linher-Melville, K. et al. Establishing a relationship between prolactin and altered fatty acid $\beta$-oxidation via carnitine palmitoyl transferase 1 in breast cancer cells. BMC Cancer 11, 56 (2011).

30. Mazzarelli, P. et al. Carnitine palmitoyltransferase I in human carcinomas: a novel role in histone deacetylation? Cancer Biol. Ther. 6, 1606-1613 (2007).

31. Grosch, S., Schiffmann, S. \& Geisslinger, G. Chain length-specific properties of ceramides. Prog. Lipid Res. 51, 50-62 (2012).

32. Ricciardi, M. R. et al. Targeting the leukemia cell metabolism by the CPT1a inhibition: functional preclinical effects in leukemias. Blood 126, 1925-1929 (2015)

33. Shao, H. J. et al. Carnitine palmitoyltransferase $1 \mathrm{~A}$ functions to repress FoxO transcription factors to allow cell cycle progression in ovarian cancer. Oncotarget 7, 3832-3846 (2016).

34. Schlaepfer, I. R. et al. Lipid catabolism via CPT1 as a therapeutic target for prostate cancer. Mol. Cancer Ther. 13, 2361-2371 (2014).

35. Schlaepfer, I. R. et al. Inhibition of lipid oxidation increases glucose metabolism and enhances 2-deoxy-2-[(18)Fffluoro-d-glucose uptake in prostate cancer mouse xenografts. Mol. Imaging Biol.: MIB: Off. Publ. Acad. Mol. Imaging 17, 529-538 (2015).

36. Pucci, S. et al. Carnitine palmitoyl transferase-1A (CPT1A): a new tumor specific target in human breast cancer. Oncotarget 7, 19982-19996 (2016).

37. Svensson, R. U. et al. Inhibition of acetyl-CoA carboxylase suppresses fatty acid synthesis and tumor growth of non-small-cell lung cancer in preclinical models. Nat. Med. 22, 1108-1119 (2016).

38. German, N. J. et al. PHD3 loss in cancer enables metabolic reliance on fatty acid oxidation via deactivation of ACC2. Mol. Cell 63, 1006-1020 (2016).

39. Casals, N. et al. Carnitine palmitoyltransferase 1C: from cognition to cancer. Prog. Lipid Res. 61, 134-148 (2016)

40. McGarry, J. D. \& Brown, N. F. The mitochondrial carnitine palmitoyltransferase system. From concept to molecular analysis. Eur. J. Biochem. 244, 1-14 (1997).

41. Yeh, C. S. et al. Fatty acid metabolism pathway play an important role in carcinogenesis of human colorectal cancers by Microarray-Bioinformatics analysis. Cancer Lett. 233, 297-308 (2006).

42. Kim, W. T. et al. Metabolic pathway signatures associated with urinary metabolite biomarkers differentiate bladder cancer patients from healthy controls. Yonsei Med. J. 57, 865-871 (2016).

43. Zaugg, K. et al. Carnitine palmitoyltransferase $1 C$ promotes cell survival and tumor growth under conditions of metabolic stress. Genes Dev. 25 1041-1051 (2011).

44. Lohse, l., Reilly, P. \& Zaugg, K. The CPT1C 5' UTR contains a repressing upstream open reading frame that is regulated by cellular energy availability and AMPK. PLOS ONE6 6, e21486 (2011).

45. Reilly, P. T. \& Mak, T. W. Molecular pathways: tumor cells co-opt the brainspecific metabolism gene CPT1C to promote survival. Clin. Cancer Res. 18, 5850-5855 (2012).

46. Sanchez-Macedo, N. et al. Depletion of the novel p53-target gene carnitine palmitoyltransferase $1 \mathrm{C}$ delays tumor growth in the neurofibromatosis type I tumor model. Cell Death Differ. 20, 659-668 (2013).

47. Carrasco, P. et al. Ceramide levels regulated by carnitine palmitoyltransferase $1 C$ control dendritic spine maturation and cognition. J. Biol. Chem. 287, 21224-21232 (2012)

48. Wu, Y., Sarkissyan, M., Mcghee, E., Lee, S. \& Vadgama, J. V. Combined inhibition of glycolysis and AMPK induces synergistic breast cancer cell killing. Breast Cancer Res. Tr. 151, 529-539 (2015).

49. Chen, Y. et al. PPARalpha regulates tumor cell proliferation and senescence via a novel target gene carnitine palmitoyltransferase 1C. Carcinogenesis $\mathbf{3 8}$, 474-483 (2017)

50. Cirillo, A. et al. High grade glioblastoma is associated with aberrant expression of ZFP57, a protein involved in gene imprinting, and of CPT1A and CPT1C that regulate fatty acid metabolism. Cancer Biol. Ther. 15, 735-741 (2014)

51. Wakamiya, T. et al. Elevated expression of fatty acid synthase and nuclear localization of carnitine palmitoyltransferase $1 \mathrm{C}$ are common among human gliomas. Neuropathol.: Off. J. Jpn. Soc. Neuropathol. 34, 465-474 (2014).

52. Guo, H. L. et al. Integrated transcriptomic analysis of distance-related field cancerization in rectal cancer patients. Oncotarget $\mathbf{8}$, 61107-61117 (2017)

53. Liu, P. P. et al. Elimination of chronic lymphocytic leukemia cells in stromal microenvironment by targeting (PT with an antiangina drug perhexiline. Oncogene 35, 5663-5673 (2016) 
54. Park, J. H. et al. Fatty acid oxidation-driven Src links mitochondrial energy reprogramming and oncogenic properties in triple-negative breast cancer. Cell Rep. 14, 2154-2165 (2016).

55. Aleshin, A. \& Finn, R. S. SRC: a century of science brought to the clinic. Neoplasia 12, 599-607 (2010).

56. Ramsay, R. R., Gandour, R. D. \& van der Leij, F. R. Molecular enzymology of carnitine transfer and transport. Biochim. Biophys. Acta 1546, 21-43 (2001)

57. Giangregorio, N., Tonazzi, A., Console, L. \& Indiveri, C. Post-translational modification by acetylation regulates the mitochondrial carnitine/acylcarnitine transport protein. Mol. Cell. Biochem. 426, 65-73 (2017).

58. Peluso, G. et al. Decreased mitochondrial carnitine translocase in skeletal muscles impairs utilization of fatty acids in insulin-resistant patients. Front. Biosci. 7, A109-A116 (2002).

59. Peluso, G. et al. Differential carnitine/acylcarnitine translocase expression defines distinct metabolic signatures in skeletal muscle cells. J. Cell. Physiol. 203, 439-446 (2005)

60. Houten, S. M. \& Wanders, R. J. A. A general introduction to the biochemistry of mitochondrial fatty acid beta-oxidation. J. Inherit. Metab. Dis. 33, 469-477 (2010).

61. Pieklik, J. R. \& Guynn, R. W. Equilibrium constants of the reactions of choline acetyltransferase, carnitine acetyltransferase, and acetylcholinesterase under physiological conditions. J. Biol. Chem. 250, 4445-4450 (1975).

62. Muoio, D. M. Metabolic inflexibility: when mitochondrial indecision leads to metabolic gridlock. Cell 159, 1253-1262 (2014).

63. Seiler, S. E. et al. Obesity and lipid stress inhibit carnitine acetyltransferase activity. J. Lipid Res. 55, 635-644 (2014).

64. Seiler, S. E. et al. Carnitine acetyltransferase mitigates metabolic inertia and muscle fatigue during exercise. Cell Metab. 22, 65-76 (2015).

65. Noland, R. C. et al. Carnitine insufficiency caused by aging and overnutrition compromises mitochondrial performance and metabolic control. J. Biol. Chem. 284, 22840-22852 (2009)

66. Muoio, D. M. et al. Muscle-specific deletion of carnitine acetyltransferase compromises glucose tolerance and metabolic flexibility. Cell. Metab. 15, 764-777 (2012)

67. Madiraju, P., Pande, S. V., Prentki, M. \& Madiraju, S. R. Mitochondrial acetylcarnitine provides acetyl groups for nuclear histone acetylation. Epigenetics 4. 399-403 (2009).

68. Wellen, K. E. et al. ATP-citrate lyase links cellular metabolism to histone acetylation. Science 324, 1076-1080 (2009).

69. Boroughs, L. K. \& DeBerardinis, R. J. Metabolic pathways promoting cancer cell survival and growth. Nat. Cell Biol. 17, 351-359 (2015).

70. Holness, M. J. \& Sugden, M. C. Regulation of pyruvate dehydrogenase complex activity by reversible phosphorylation. Biochem. Soc. Trans. 31, 1143-1151 (2003). (Pt 6).

71. Corbet, $\mathrm{C}$. et al. Acidosis drives the reprogramming of fatty acid metabolism in cancer cells through changes in mitochondrial and histone acetylation. Cell Metab. 24, 311-323 (2016).

72. McDonnell, E. et al. Lipids reprogram metabolism to become a major carbon source for histone acetylation. Cell Rep. 17, 1463-1472 (2016).

73. Krol, J., Loedige, I. \& Filipowicz, W. The widespread regulation of microRNA biogenesis, function and decay. Nat. Rev. Genet. 11, 597-610 (2010).

74. Garzon, R., Marcucci, G. \& Croce, C. M. Targeting microRNAs in cancer: rationale, strategies and challenges. Nat. Rev. Drug Discov. 9, 775-789 (2010).

75. Li, Z. H. \& Rana, T. M. Therapeutic targeting of microRNAs: current status and future challenges. Nat. Rev. Drug Discov. 13, 622-638 (2014).

76. Bartel, D. P. MicroRNAs: genomics, biogenesis, mechanism, and function (Reprinted from Cell 116, 281-297 (2004)). Cell 131, 11-29 (2007).

77. He, L. \& Hannon, G. J. MicroRNAs: Small RNAs with a big role in gene regulation (vol 5, pg 522 2004). Nat. Rev. Genet. 5, 522 (2004). -+.

78. Ambros, V. The functions of animal microRNAs. Nature 431, 350-355 (2004)
79. Volinia, S. et al. A microRNA expression signature of human solid tumors defines cancer gene targets. Proc. Natl. Acad. Sci. USA 103, 2257-2261 (2006).

80. Zhang, B. H. \& Farwell, M. A. microRNAs: a new emerging class of players for disease diagnostics and gene therapy. J. Cell. Mol. Med. 12, 3-21 (2008).

81. Rottiers, V. \& Naar, A. M. MicroRNAs in metabolism and metabolic disorders. Nat. Rev. Mol. Cell Biol. 13, 239-250 (2012).

82. Chen, B. et al. Roles of microRNA on cancer cell metabolism. J. Transl. Med. 10, 228 (2012)

83. Lagos-Quintana, M. et al. Identification of tissue-specific microRNAs from mouse. Curr. Biol. 12, 735-739 (2002)

84. Elmen, J. et al. LNA-mediated microRNA silencing in non-human primates. Nature 452, 896-U10 (2008)

85. Esau, C. et al. miR-122 regulation of lipid metabolism revealed by in vivo antisense targeting. Cell Metab. 3, 87-98 (2006).

86. Iliopoulos, D., Drosatos, K., Hiyama, Y., Goldberg, I. J. \& Zannis, V. I. MicroRNA 370 controls the expression of microRNA-122 and Cpt1alpha and affects lipid metabolism. J. Lipid Res. 51, 1513-1523 (2010).

87. Najafi-Shoushtari, S. H. et al. MicroRNA-33 and the SREBP Host Genes Cooperate to Control Cholesterol Homeostasis. Science 328, 1566-1569 (2010)

88. Rayner, K. J. et al. MiR-33 contributes to the regulation of cholestero homeostasis. Science 328, 1570-1573 (2010).

89. Eichner, L. J. et al. miR-378(*) mediates metabolic shift in breast cancer cells via the PGC-1beta/ERRgamma transcriptional pathway. Cell Metab. 12, 352-361 (2010)

90. Carrer, M. et al. Control of mitochondrial metabolism and systemic energy homeostasis by microRNAs 378 and 378*. Proc. Natl. Acad. Sci. USA 109 15330-15335 (2012).

91. Esau, C. et al. MicroRNA-143 regulates adipocyte differentiation. J. Biol. Chem. 279, 52361-52365 (2004)

92. Shirasaki, T. et al. MicroRNA-27a regulates lipid metabolism and inhibits hepatitis C virus replication in human hepatoma cells. J. Virol. 87, 5270-5286 (2013)

93. Nakanishi, N. et al. The up-regulation of microRNA-335 is associated with lipid metabolism in liver and white adipose tissue of genetically obese mice. Biochem. Biophys. Res. Commun. 385, 492-496 (2009).

94. Xu, P. Z., Vernooy, S. Y., Guo, M. \& Hay, B. A. The Drosophila microRNA mir-14 suppresses cell death and is required for normal fat metabolism. Curr. Biol. 13, 790-795 (2003)

95. Cui, M et al. MiR-205 modulates abnormal lipid metabolism of hepatoma cells via targeting acyl-CoA synthetase long-chain family member 1 (ACSL1) mRNA. Biochem. Biophys. Res. Commun. 444, 270-275 (2014).

96. Menendez, J. A., Decker, J. P. \& Lupu, R. In support of fatty acid synthase (FAS) as a metabolic oncogene: Extracellular acidosis acts in an epigenetic fashion activating FAS gene expression in cancer cells. J. Cell. Biochem. 94, 1-4 (2005).

97. Fernandez-Hernando, C., Suarez, Y., Rayner, K. J. \& Moore, K. J. MicroRNAs in lipid metabolism. Curr. Opin. Lipidol. 22, 86-92 (2011).

98. Esser, V. et al. Inhibitors of mitochondrial carnitine palmitoyltransferase-l limit the action of proteases on the enzyme-isolation and partial amino-acidanalysis of a truncated form of the rat-liver isozyme. J. Biol. Chem. 268, 5810-5816 (1993).

99. Yamazaki, N., Shinohara, Y., Shima, A. \& Terada, H. High expression of a novel carnitine palmitoyltransferase-l like protein in rat brown adipose-tissue and heart-isolation and characterization of its Cdna clone. FEBS Lett. 363, 41-45 (1995)

100. Ramsay, R. R., Gandour, R. D. \& van der Leij, F. R. Molecular enzymology of carnitine transfer and transport. Bba-Protein Struct. $M$. 1546, 21-43 (2001)

101. Bonnefont, J. P. et al. Carnitine palmitoyltransferases 1 and 2: biochemical, molecular and medical aspects. Mol. Asp. Med. 25, 495-520 (2004).

102. Jogl, G., Hsiao, Y. S. \& Tong, L. Structure and function of carnitine acyltransferases. Ann. NY Acad. Sci. 1033, 17-29 (2004). 